\title{
QRS Fragmentation in Preserved Ejection Fraction Heart Failure: Functional Insights, Pathological Correlates, and Prognosis
}

\section{Kuo-Tzu Sung}

Mackay Memorial Hospital

Sheng-Hsiung Chang

Mackay Memorial Hospital

Po-Ching Chi

Zhongmei Hospital, Taiwan

Shih-Chieh Chien

Mackay Memorial Hospital

Chi-In Lo

Mackay Memorial Hospital

Chao-Feng Lin

Mackay Memorial Hospital

Wen-Hung Huang

Mackay Memorial Hospital

Chun-Ho Yun

Mackay Memorial Hospital

Cheng-Ting Tsai

Mackay Memorial Hospital

Cheng-Huang Su

Mackay Memorial Hospital

Charles Jia-Yin Hou

Mackay Memorial Hospital

Hung-I Yeh

Mackay Memorial Hospital

Chin-Ho Tsao

Mackay Memorial Hospital

Jen-Yuan Kuo ( $\nabla$ jykuo5813@gmail.com )

Mackay Memorial Hospital

Chung-Lieh Hung

Mackay Memorial Hospital 


\section{Research Article}

Keywords: fragmented QRS complex, heart failure preserved ejection fraction, mortality, QRS duration Posted Date: October 25th, 2021

DOl: https://doi.org/10.21203/rs.3.rs-943276/v1

License: (c) (i) This work is licensed under a Creative Commons Attribution 4.0 International License. Read Full License 


\section{Abstract}

Backgrounds: Fragmented QRS (fQRS) morphology as a surrogate marker of the possible presence of myocardial scarring has been shown to confer a higher risk in patients with reduced ejection fraction heart failure. We aimed to investigate the pathophysiological correlates and prognostic implications of fQRS in patients with preserved ejection fraction heart failure (HFpEF).

Methods and Results: We consecutively studied 960 patients with HFpEF (76.4 \pm 12.7 years, men: $37.2 \%)$. fQRS was assessed using a body surface electrocardiogram during hospitalization. QRS morphology was available and classified into three categories among $960 \mathrm{HFpEF}$ subjects as non-fQRS, inferior fQRS, and anterior/lateral fQRS groups. Despite comparable clinical features in most baseline demographics among the three fQRS categories, anterior/lateral fQRS showed significantly higher BNP/troponin levels (both $p<0.001$ ), with both the inferior and anterior/lateral fQRS HFpEF groups demonstrating a higher degree of unfavorable cardiac remodeling, greater extent of myocardial perfusion defect, and slower coronary flow phenomenon (all $p<0.05$ ). Anterior/lateral fQRS HFpEF patients exhibited significantly altered cardiac structure/function and more impaired diastolic indices (all $p<0.05$ ). During a median of 657 days follow-up, the presence of anterior/lateral fQRS conferred a doubled HF re-admission risk (adjusted HR: 1.90, p<0.001), with both inferior and anterior/lateral fQRS having a higher risk of cardiovascular and all-cause death (all $p<0.05$ ) by using Cox regression models.

Conclusions: The presence of $\mathrm{fQRS}$ in HFpEF was associated with more extensive myocardial perfusion defects and worsened mechanics, which possibly denotes a more severe involvement of cardiac damage. Early recognition in such HFpEF patients likely benefits from targeted therapeutic interventions.

\section{Introduction}

Fragmented QRS (fQRS) refers to various QRS morphologies that are typically less than $120 \mathrm{~ms}$ in duration in the presence or absence of the Q-wave on body surface 12-lead electrocardiography (ECG). It is characterized by an $\mathrm{R}^{\prime}$ or notching in the nadir of the $S$ wave, and there can also be more than one $\mathrm{R}^{\prime}$ in two contiguous leads corresponding to a major coronary artery territory $(1,2)$. fQRS complexes are new ECG signals associated with varied conduction abnormalities and are assumed to originate from the delay in peri-infarct conduction due to myocardial scarring or necrosis $(1,2)$. This specific ECG morphology is observed in patients with coronary artery diseases, cardiomyopathies, structural heart diseases, heart rhythm disturbances, and cardiac sarcoidosis (3-5). Several studies have shown that the presence of $\mathrm{fQRS}$ is a predictor of sudden cardiac death in patients with cardiomyopathy and heart failure (HF) $(4,6)$. Furthermore, it has been shown to be a prognostic marker of cardiac events and mortality in patients with coronary artery disease $(C A D)(7,8)$, and a predictor of dysrhythmia in patients with Brugada syndrome (9).

As a clinical HF phenotype accompanied by a high clinical co-morbidity burden (i.e., coronary heart disease or hypertrophy from hypertension) with unfavorable myocardial remodeling, patients with heart 
failure with preserved ejection fraction (HFpEF) may theoretically have a higher chance of developing a longer QRS duration (10). However, reports investigating the prevalence and significance of fQRS in HFpEF or the general population were either small in sample size or with study limitations (11-13).

In this study, we sought to investigate the prevalence and long-term prognostic significance of fQRS and QRS duration in patients with HFpEF.

\section{Methods}

\section{Study Subjects}

Our current study was retrospective in the study design. The study workflow including setting, study participants selection, and exclusion criteria have been published previously and further detailed (14) (Supplemental Figure 1). In brief, we investigated 1120 consecutively discharged HFpEF patients aged > 20 years who were discharged with a main clinical diagnosis of discharge HF from a tertiary medical center located in Northern Taiwan (from March 2012 to December 2014). Medical information regarding the diagnosis, medical history, complete 12-lead ECG, and laboratory data were all obtained in study participants. A total of $960 \mathrm{HFpEF}$ patients had complete body surface ECG information for fQRS categorization of in the current analysis. This study was conducted following the guidelines of the Declaration of Helsinki and was approved by the Mackay Memorial Hospital Institutional Review Board (15MMHIS015). All patients provided written consent to participate in our study.

\section{Definition of QRS Fragmentation (fQRS)}

Standard 12-lead ECGs were collected and analyzed. The fQRS in the current study was limited to ECGs with QRS duration $<120 \mathrm{~ms}$ and was defined by the presence of various RSR patterns, including the presence of an additional $R$ wave $(R)$, a notching of the $R$ wave, a notching in the nadir of the $S$ wave, or the presence of more than one $\mathrm{R}$ in at least two contiguous leads, corresponding to a specific and major coronary artery territory (7). Bundle branch block (BBB) were excluded from the definition of fQRS.

Furthermore, when notching was confined to the terminal QRS accompanied by a J-point elevation of at least $0.1 \mathrm{mV}$, it was classified as early repolarization rather than fQRS (15).

fQRS was then further categorized by anatomic locations and coronary territory distributions. fQRS was classified into anterior (leads V1-V5), lateral (leads I, aVL, and V6), or inferior (leads II, III, and aVF) (16). Among the 1120 admitted patients with HFpEF, 960 had ECG-defined QRS morphology data available and were classified into the three categories: 657 non-fQRS, 220 inferior fQRS, and 83 subjects with fQRS on anterior or lateral leads (anterior/lateral). We also classified our study participants into 2 groups according to QRS duration cutoff: $\leq 110 \mathrm{~ms}$ and those $>110 \mathrm{~ms}$.

\section{Echocardiography}

Comprehensive echocardiography was performed within the first 3 days of HF admission. Twodimensional cine loops and Doppler images are acquired in three consecutive heart beats during the 
hospital stay or on arrival at the emergency department. Our current study mainly focused on parameters of left ventricular (LV) structure (wall thickness, internal dimension, LV mass index, LV end-diastolic and end-systolic volume) and geometry as recommended by a standardized protocol, with LVEF determined by using biplane Simpson's method (17). Right ventricular (RV) structure/function (RV end-diastolic [RV EDA] and end-systolic area [RV ESA]) and RV function (fractional area change: RVFAC, as \%) were also determined. LV diastolic function including mitral E/A ratio, deceleration time, interventricular relaxation time, early mitral annulus tissue Doppler velocity e' and s', LV filling E divided by e' (E/e'), and bi-atrial (right [RA] and left atrium [LA]) volume index were all analyzed and quantified using contemporary guidelines. (18).

\section{SPECT and Coronary Flow Angiography}

Overall, 413 out of 960 (43.0\%) HFpEF patients underwent coronary angiography due to known CAD (including myocardial infarction) or symptom-driven indications. Among 326 out of 960 study participants with CAD, 210 (64.4\% of all known CAD population) had post-PCl radiopharmaceutical SPECT (technetium-99m sestamibi) myocardial scan data available within 6 months of the study enrollment date. SPECT myocardial perfusion burden was quantified using quantitative gated SPECT (QGS) and quantitative perfusion SPECT (QPS) software. Among the symptom-driven 634 non-CAD participants, 203 (32.0\%) underwent coronary angiography. Coronary flow grades for the symptom-driven 203 non-CAD participants were assessed using the corrected TIMI frame count (CTFC) method, which represents the total number of cine-frames required for contrast to first reach standard distal coronary landmarks. The coronary slow flow in the current study was defined as TIMI frame count $>27$ frames in the current study (19).

\section{Clinical Outcomes}

The primary clinical outcome measures of the current study included pre-specified clinical endpoints: hospitalization for heart failure (hHF), cardiovascular (CV) mortality, all-cause mortality, and the composite endpoints of hHF and any death during follow-up after the study indexed date. The study index date was defined as the date after establishment of HFpEF diagnosis. These clinical endpoints were further adjudicated by two experienced cardiologists (SCC and CLH), mainly based on the extracted EDC information.

\section{Statistical Analysis}

Comparisons of the continuous variables between the two groups were performed using an unpaired 2tailed t test, with nominal/categorical variables compared by a chi-square analysis or Fisher's exact test, as appropriate. Continuous variables among the different clinical categories of more than 3 groups were performed using a one-way analysis of variance (one-way ANOVA) or Kruskal-Wallis test with post hoc paired comparisons as appropriate.

Multivariate Cox regression models were used to examine the associations of region-specific fQRS (NonfQRS as reference group) with clinical endpoints, including heart failure hospitalization (hHF), 
cardiovascular (CV) mortality, all-cause mortality, and the composite endpoints of hHF and any death. Individual hazard ratio (HR) and correspondent 95\% confidence intervals (95\% Cl). Key clinical covariates including age, sex, body size, medical histories of hypertension, diabetes, hyperlipidemia treatment, atrial fibrillation, stroke, known coronary artery disease, CVA, eGFR, and LVEF from echocardiography data served as confounders. Kaplan-Meier failure analysis and the log-rank test for $\mathrm{p}$-trend across different fQRS strata were performed for determining the incidence of hHF, CV death, all-cause mortality, and composite endpoint. Statistical significance was set at $p<0.05$ using STATA software (version 13.1).

\section{Results}

\section{Baseline Characteristics}

Among the original $1120 \mathrm{HFpEF}$ patients enrolled, we identified total $960 \mathrm{HFpEF}$ patients $(76.4 \pm 12.7$ years, men: $37.2 \%)$ with fQRS classification available as non-fQRS (68.4\%), inferior fQRS (22.9\%), and anterior/lateral fQRS (8.7\%) without overt BB and after application of our exclusion criteria (Table 1). Compared to those classified as non-fQRS, patients with inferior fQRS group had higher systolic blood pressure and were more likely to have diabetes mellitus; patients with anterior/lateral fQRS had higher serum potassium level and were more likely to have atrial fibrillation (Table 1), while other baseline demographics were comparable. We also observed graded and significantly higher BNP and troponin I levels across the three categories (non-fQRS, inferior fQRS, anterior/later fQRS, BNP: 554 vs 581 vs 893 $\mathrm{pg} / \mathrm{mL}$; troponin-l: 0.04 vs 0.04 vs 0.06 as median [IQR] ranges, aall trend $p<0.01$ ). Baseline demographics according to QRS duration strata cutoff: $110 \mathrm{~ms}$ ) were detailed in Table S1. 
Table 1

Baseline characteristics of study subjects categorized by the location of fragmented QRS

$\begin{array}{lllll}\text { Fragmented QRS Groups } & \text { Non-fQRS } & \text { Inferior fQRS } & \begin{array}{l}\text { Anterior/lateral } \\ \text { fQRS }\end{array} & \begin{array}{l}\text { pvalue } \\ \text { (ANOVA) }\end{array} \\ & (\mathrm{N}=657) & (\mathrm{N}=220) & (\mathrm{N}=83) & \end{array}$

Demographics

\begin{tabular}{|c|c|c|c|c|}
\hline Age & $76.7 \pm 12.8$ & $75.8 \pm 12.5$ & $75.6 \pm 12.6$ & 0.41 \\
\hline Gender, male (\%) & $238(36.2 \%)$ & $86(39.1 \%)$ & $33(39.8 \%)$ & 0.52 \\
\hline 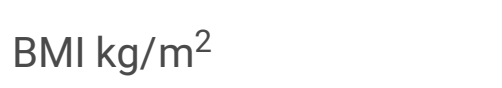 & $24.6 \pm 5.5$ & $25.3 \pm 5.7$ & $24.4 \pm 11.2$ & 0.04 \\
\hline SBP, mmHg & $139.5 \pm 32.3$ & $146.1 \pm 32.8^{*}$ & $138.8 \pm 34.3$ & 0.02 \\
\hline $\mathrm{DBP}, \mathrm{mmHg}$ & $72.6 \pm 17.5$ & $74.4 \pm 18.8$ & $73.5 \pm 19.8$ & 0.41 \\
\hline $\mathrm{HR}$, bpm & $90.4 \pm 2.3$ & $88.8 \pm 19.3$ & $94.7 \pm 4.9$ & 0.09 \\
\hline \multicolumn{5}{|l|}{ History, n (\%) } \\
\hline Prior heart failure & $362(55.1 \%)$ & $118(53.6 \%)$ & $46(55.4 \%)$ & 0.89 \\
\hline Hypertension & $469(71.4 \%)$ & $171(77.7 \%)$ & $61(73.5 \%)$ & 0.22 \\
\hline Diabetes mellitus & $315(47.9 \%)$ & $127(58.0 \%)$ & $39(47.0 \%)$ & 0.03 \\
\hline Coronary artery disease & $215(32.7 \%)$ & $76(34.7 \%)$ & $34(41.0 \%)$ & 0.31 \\
\hline ESRD/Hemodialysis & $72(11.0 \%)$ & $26(11.8 \%)$ & $16(19.3 \%)$ & 0.16 \\
\hline Hyperlipidemia treatment & $118(18.0 \%)$ & $47(21.4 \%)$ & $12(14.5 \%)$ & 0.31 \\
\hline Stroke & $111(16.9 \%)$ & $39(17.7 \%)$ & $17(20.5 \%)$ & 0.71 \\
\hline Atrial fibrillation & $132(20.1 \%)$ & $36(16.4 \%)$ & $24(28.9 \%) \dagger$ & 0.09 \\
\hline PAD & $44(6.7 \%)$ & $16(7.3 \%)$ & $5(6.0 \%)$ & 0.91 \\
\hline \multicolumn{5}{|l|}{ Laboratory } \\
\hline Fasting glucose, mg/dl & $170.1 \pm 108.1$ & $184.5 \pm 114.6$ & $192.4 \pm 131.3$ & 0.09 \\
\hline eGFR, $\mathrm{ml} / \mathrm{min} / 1.73 \mathrm{~m}^{2}$ & $42.1 \pm 32.9$ & $39.0 \pm 32.9$ & $39.0 \pm 32.4$ & 0.41 \\
\hline
\end{tabular}

Data are expressed as mean \pm SD or percentage. ACEl, angiotensin-converting enzyme inhibitor; ARB, angiotensin II-receptor blocker; BNP, B-type natriuretic peptide; BMI, body mass index; DBP, diastolic blood pressure; eGFR, estimated glomerular filtration rate; ESRD, end-stage renal disease; HR, heart rate; LVEF, left ventricular ejection fraction; PAD, peripheral arterial disease; SBP, systolic blood pressure.

* $p<0.05$ vs Non-fQRS; $\uparrow p<0.05$ vs Inferior fQRS.

a Troponin-I and BNP are expressed as median [25th percentile, 75th percentile] 


\begin{tabular}{|c|c|c|c|c|}
\hline Fragmented QRS Groups & $\begin{array}{l}\text { Non-fQRS } \\
(\mathrm{N}=657)\end{array}$ & $\begin{array}{l}\text { Inferior fQRS } \\
(\mathrm{N}=220)\end{array}$ & $\begin{array}{l}\text { Anterior/lateral } \\
\text { fQRS } \\
(\mathrm{N}=83)\end{array}$ & $\begin{array}{l}\text { pvalue } \\
\text { (ANOVA) }\end{array}$ \\
\hline$A L T, u / l$ & $52.7 \pm 108.0$ & $78.6 \pm 200.4$ & $87.3 \pm 261.1$ & 0.03 \\
\hline Serum sodium, $\mathrm{mmol} / \mathrm{L}$ & $131.0 \pm 8.4$ & $129.9 \pm 7.7$ & $129.2 \pm 9.2$ & 0.10 \\
\hline $\begin{array}{l}\text { Serum potassium, } \\
\mathrm{mmol} / \mathrm{L}\end{array}$ & $5.2 \pm 1.2$ & $5.4 \pm 1.3$ & $5.6 \pm 1.3^{\star}$ & 0.007 \\
\hline $\mathrm{BNP}, \mathrm{pg} / \mathrm{mL}(\mathrm{n}=917)^{\mathrm{a}}$ & $\begin{array}{l}554[256 \\
1170]\end{array}$ & $\begin{array}{l}581[246 \\
1330]\end{array}$ & $893[478,2740]^{\star \star}$ & $<0.001$ \\
\hline Troponin I $(\mathrm{n}=887)^{\mathrm{a}}$ & $\begin{array}{l}0.03[0.02, \\
0.1]\end{array}$ & $\begin{array}{l}0.04[0.02 \\
0.12]\end{array}$ & $0.06[0.03,0.23]^{\star \dagger}$ & 0.008 \\
\hline QRS duration, ms & $92.3 \pm 15.0$ & $94.2 \pm 15.3$ & $97.8 \pm 17.3^{*}$ & 0.005 \\
\hline NYHA Fc & & & & 0.061 \\
\hline$\leq \|$ & $14.3 \%$ & $14.5 \%$ & $12.0 \%$ & \\
\hline III & $62.2 \%$ & $60.0 \%$ & $49.4 \%$ & \\
\hline IV & $23.4 \%$ & $25.4 \%$ & $38.6 \%$ & \\
\hline \multicolumn{5}{|l|}{ Measurement } \\
\hline LVEF, \% & $64.7 \pm 6.3$ & $64.8 \pm 6.7$ & $64.1 \pm 7.1$ & 0.66 \\
\hline $\begin{array}{l}\text { Prognostic nutritional } \\
\text { score }\end{array}$ & $41.3 \pm 9.1$ & $41.3 \pm 8.9$ & $40.0 \pm 9.4$ & 0.43 \\
\hline \multicolumn{5}{|l|}{ Medications, n (\%) } \\
\hline ACEI/ARB & $220(33.5 \%)$ & $83(37.7 \%)$ & $27(32.5 \%)$ & 0.45 \\
\hline Beta-blocker & $133(20.2 \%)$ & $48(21.8 \%)$ & $14(16.8 \%)$ & 0.73 \\
\hline Aldosterone antagonists & $105(16.0 \%)$ & $39(17.7 \%)$ & $11(13.3 \%)$ & 0.61 \\
\hline Digoxin & $34(5.2 \%)$ & $14(6.4 \%)$ & $7(8.4 \%)$ & 0.43 \\
\hline Diuretics & $297(45.2 \%)$ & $105(47.7 \%)$ & $28(33.7 \%)$ & 0.05 \\
\hline \multicolumn{5}{|c|}{$\begin{array}{l}\text { Data are expressed as mean } \pm \text { SD or percentage. ACEI, angiotensin-converting enzyme inhibitor; ARB, } \\
\text { angiotensin II-receptor blocker; BNP, B-type natriuretic peptide; BMI, body mass index; DBP, diastolic } \\
\text { blood pressure; eGFR, estimated glomerular filtration rate; ESRD, end-stage renal disease; HR, heart } \\
\text { rate; LVEF, left ventricular ejection fraction; PAD, peripheral arterial disease; SBP, systolic blood } \\
\text { pressure. }\end{array}$} \\
\hline \multicolumn{5}{|c|}{${ }^{*} p<0.05$ vs Non-fQRS; $\uparrow p<0.05$ vs Inferior fQRS. } \\
\hline
\end{tabular}




\section{Cardiac Structure and Function}

Compared to those classified as non-fQRS, HFpEF patients with anterior/lateral fQRS had a substantially larger LV EDVi $(48.9 \pm 17.0 \mathrm{~mL}$ vs $44.2 \pm 13.8 \mathrm{~mL}, p<0.05)$, greater LV mass-to-volume ratio $(2.21 \pm 0.68$ vs. $2.19 \pm 0.84, p<0.05)$, and had significantly lower myocardial relaxation velocity TDI-e' $(4.8 \pm 1.9$ vs $5.7 \pm 1.8$ $\mathrm{cm} / \mathrm{s}, p<0.05)$. Both patient groups with inferior and anterior/lateral fQRS presented with a significantly larger LV mass (174.9 \pm 60.7 and $180.2 \pm 75.1 \mathrm{gm}$ vs. $162.8 \pm 58.6 \mathrm{gm}$, trend $p=0.004)$, with those with anterior/lateral fQRS had significantly larger indexed LV mass and were more likely to have LVH compared to non-fQRS (39.6\% and $49.4 \%$ vs. $34.7 \%$, trend $p=0.027)$. Furthermore, compared to those classified as non-fQRS/inferior fQRS, HFpEF patients with anterior/lateral fQRS showed a substantially lower myocardial systolic velocity TDI-s'(trend $p=0.001)$, higher LV filling E/ $\mathrm{e}^{\prime}(20.4 \pm 9.8$ vs $16.1 \pm 7.0$ and $16.5 \pm 7.1$, trend $p<0.001)$, higher TR velocity $(3.2 \pm 0.6 \mathrm{~m} / \mathrm{s}$ vs $3.0 \pm 0.4$ and $3.0 \pm 0.4 \mathrm{~m} / \mathrm{s}$, trend $p=0.010)$, larger RA/LA indexed volumes ( both trend $p<0.05$; LA indexed volume: $33.0 \pm 15.0 \mathrm{~mL} / \mathrm{m}^{2}$ vs $28.3 \pm 13.3$ and $28.0 \pm 11.9 \mathrm{~mL} / \mathrm{m}^{2}$, trend $p=0.010$ ), and a greater RV end-diastolic/systolic area (both trend $p<$ 0.001). (Table 2) In addition, compared to non-fQRS, those presenting with anterior/lateral fQRS had significantly lower RVFAC $(p=0.015)$. Overall, more unfavorable cardiac structural and functional indices were found in those presenting wider $(>110 \mathrm{~ms})$ compared to those with smaller QRS duration $(\leq 110 \mathrm{~ms})$, though these differences were less prominent compared to fQRS strata (Table S2). 
Table 2

The LV/RV structure and function in non-fQRS, inferior fQRS, and anterior/lateral fQRS groups

$\begin{array}{lllll}\text { Fragmented QRS Groups } & \text { Non-fQRS } & \begin{array}{l}\text { Inferior } \\ \text { fQRS }\end{array} & \begin{array}{l}\text { Anterior/lateral } \\ \text { fQRS }\end{array} & \begin{array}{l}\text { pvalue } \\ \text { (ANOVA) }\end{array} \\ & (\mathrm{N}=657) & (\mathrm{N}=220) & (\mathrm{N}=83) & \end{array}$

\section{Structure}

\begin{tabular}{lllll} 
Septal wall thickness, $\mathrm{mm}$ & $9.9 \pm 2.0$ & $10.2 \pm 2.0$ & $10.4 \pm 1.9$ & 0.015 \\
\hline $\begin{array}{l}\text { Posterior wall thickness, } \\
\mathrm{mm}\end{array}$ & $10.0 \pm 2.0$ & $10.3 \pm 2.0$ & $10.3 \pm 1.9$ & 0.039 \\
\hline LV internal dimension, $\mathrm{mm}$ & $46.3 \pm 6.1$ & $47.0 \pm 6.2$ & $47.7 \pm 6.7$ & 0.080 \\
\hline LV EDV index, $\mathrm{mL}$ & $44.2 \pm 13.8$ & $45.2 \pm 15.5$ & $48.9 \pm 17.0^{\star}$ & 0.028 \\
\hline LV ESV index, $\mathrm{mL}$ & $15.0 \pm 8.7$ & $15.1 \pm 9.9$ & $17.3 \pm 11.8$ & 0.120 \\
\hline LVEF, \% & $67.9 \pm 13.6$ & $69.2 \pm 14.2$ & $67.6 \pm 15.4$ & 0.450 \\
\hline LV mass, gm & $162.8 \pm 58.6$ & $174.9 \pm 60.7^{*}$ & $180.2 \pm 75.1^{*}$ & 0.004 \\
\hline LV mass index, gm/m² & $94.2 \pm 30.8$ & $99.4 \pm 32.7$ & $105.3 \pm 39.5^{\star}$ & 0.004 \\
\hline Presence of $\mathrm{LVH}(\%)$ & $216(32.9 \%)$ & $84(38.2 \%)$ & $39(47.0 \%)$ & 0.027 \\
\hline LV M/V ratio & $2.2 \pm 0.8$ & $2.3 \pm 1.0$ & $2.2 \pm 0.7^{*}$ & 0.684
\end{tabular}

LV function

\begin{tabular}{|lllll}
\hline Mitral E/A ratio & $1.2 \pm 1.5$ & $1.1 \pm 0.8$ & $1.2 \pm 0.8$ & 0.370 \\
\hline DT, ms & $210.8 \pm 77.3$ & $211.1 \pm 74.8$ & $206.7 \pm 79.9$ & 0.892 \\
\hline IVRT, ms & $85.9 \pm 33.4$ & $87.5 \pm 30.5$ & $91.5 \pm 34.3$ & 0.312 \\
\hline TDI-e' & $5.7 \pm 1.8$ & $5.4 \pm 1.8$ & $4.8 \pm 1.9^{*}$ & 0.001 \\
\hline TDI-s' & $5.7 \pm 1.4$ & $5.6 \pm 1.3$ & $5.0 \pm 1.3^{*}+$ & 0.001 \\
\hline Mitral E/TDI-e' & $16.1 \pm 7.0$ & $16.5 \pm 7.1$ & $20.4 \pm 9.8^{*}+$ & $<0.001$ \\
\hline TR Velocity, cm/s & $3.0 \pm 0.4$ & $3.0 \pm 0.5$ & $3.2 \pm 0.6^{*}+$ & 0.010
\end{tabular}

$\mathrm{RV}$ structure and function

Data are expressed as mean \pm SD or percentage. All expressions are listed in Table 1 .

* $p<0.05$ vs Non-fQRS; † $p<0.05$ vs Inferior fQRS.

LV, left ventricular; RV, right ventricular; EDV, end-diastolic volume; EDA, end-diastolic area; ESA, endsystolic area; FAC, fractional area change; ESV, end-systolic volume; LVEF, left ventricular ejection fraction; LVH, left ventricular hypertrophy; LV, left ventricular mass to volume ratio; DT, deceleration time; IVRT, interventricular relaxation time; LA, left atrial; RA, right atrial; TDI, tissue Doppler imaging; $\mathrm{TR}$, tricuspid regurgitation. 


\begin{tabular}{|c|c|c|c|c|}
\hline Fragmented QRS Groups & $\begin{array}{l}\text { Non-fQRS } \\
(\mathrm{N}=657)\end{array}$ & $\begin{array}{l}\text { Inferior } \\
\text { fQRS } \\
(\mathrm{N}=220)\end{array}$ & $\begin{array}{l}\text { Anterior/lateral } \\
\text { fQRS } \\
(\mathrm{N}=83)\end{array}$ & $\begin{array}{l}\text { pvalue } \\
\text { (ANOVA) }\end{array}$ \\
\hline $\mathrm{RV} E D A, \mathrm{~cm}^{2}$ & $30.5 \pm 13.1$ & $31.9 \pm 13.5$ & $37.3 \pm 16.3^{\star}+$ & $<0.001$ \\
\hline RV ESA, $\mathrm{cm}^{2}$ & $16.1 \pm 8.7$ & $16.9 \pm 9.0$ & $20.9 \pm 11.9 *+$ & $<0.001$ \\
\hline RV FAC, \% & $48.4 \pm 7.9$ & $47.9 \pm 8.4$ & $45.5 \pm 8.4^{*}$ & 0.015 \\
\hline \multicolumn{5}{|l|}{ Atrial structure } \\
\hline LA Volume index, $\mathrm{mL} / \mathrm{m}^{2}$ & $28.3 \pm 13.3$ & $28.0 \pm 11.9$ & $33.0 \pm 15.0 *+$ & 0.010 \\
\hline RA Volume index, $\mathrm{mL} / \mathrm{m}^{2}$ & $22.9 \pm 14.8$ & $22.4 \pm 20.3$ & $28.3 \pm 18.2^{\star *}+$ & 0.022 \\
\hline \multicolumn{5}{|c|}{ Data are expressed as mean \pm SD or percentage. All expressions are listed in Table 1} \\
\hline \multicolumn{5}{|c|}{${ }^{*} p<0.05$ vs Non-fQRS; $\dagger p<0.05$ vs Inferior $\mathrm{fQRS}$} \\
\hline \multicolumn{5}{|c|}{$\begin{array}{l}\text { LV, left ventricular; RV, right ventricular; EDV, end-diastolic volume; EDA, end-diastolic area; ESA, end- } \\
\text { systolic area; FAC, fractional area change; ESV, end-systolic volume; LVEF, left ventricular ejection } \\
\text { fraction; LVH, left ventricular hypertrophy; LV, left ventricular mass to volume ratio; DT, deceleration } \\
\text { time; IVRT, interventricular relaxation time; LA, left atrial; RA, right atrial; TDI, tissue Doppler imaging; } \\
\text { TR, tricuspid regurgitation. }\end{array}$} \\
\hline
\end{tabular}

\section{Despite comparable CAD prevalence, HFpEF patients categorized into inferior fQRS and anterior/lateral} fQRS had a graded increase in post-stress total myocardial perfusion defect when compared to the nonfQRS group (Figure $1 \mathrm{~A}$, trend $p=0.001$ ). By examining individual myocardial perfusion defects according to coronary artery territories, HFpEF patients categorized into anterior/lateral fQRS were more likely to have fixed myocardial perfusion defects from the LAD/LCX coronary territory compared to the RCA territory $(40 \%, 40 \%$ vs. $13.3 \%)$, with those HFpEF patients categorized into inferior fQRS tend to have fixed perfusion defects close to LCX/RCA coronary territory compared to LAD (38.1\%, 33.3\% vs. $19 \%)$. HFpEF classified as non-fQRS was less likely to present myocardial perfusion defects on three coronary arterial territories (all <20\%) (Figure 1B-D).

Illustrations of myocardial perfusion deficit by SPECT are displayed in Figure 2A-C. The burden of myocardial perfusion defect was inversely correlated with TDI-s' $(r=-0.15, p=0.02)$, showing a borderline reverse relationship with TDI-e' $(r=-0.11, p=0.08)$, with a non-significant positive relationship with E/TDI$\mathrm{e}^{\prime}(\mathrm{r}=0.05, p=0.46)$ (Figure 2D-F). Overall, 67 out of 203 (33\%) symptom-driven HFpEF patients did not have a significant CAD manifested slow flow phenomenon, with a graded and significantly higher proportion observed across non-fQRS, inferior fQRS, and anterior/lateral fQRS $(28 \%, 50 \%$, and $83.3 \%$, respectively, $X^{2}<0.001$ ) (Figure $3 A$ ). Interestingly, substantially lower myocardial TDI-e' and TDI-s' were associated with a higher LV filling pressure (E/TDI-e') in those HFpEF patients without CAD manifesting slow coronary flow (Figure 3B-D). 


\section{Clinical Outcomes}

During a median follow-up of 657 days (IQR: 70-1274 days), 314 patients (32.7\%) were hospitalized for HF, 178 (18.5\%) experienced CV death, 337 (35.1\%) experienced all-cause death, and $585(60.9 \%)$ had composite events of HF hospitalization and mortality irrespective of any cause. A Cox proportional hazard regression analysis for adverse events is shown in Figure 4 and Table S3. By using non-fQRS as the reference group, the presence of anterior/lateral fQRS was associated with a higher risk of HHF (adjusted HR: 1.90 [1.34, 2.69], $p<0.001$ ), with the presence of both inferior fQRS and anterior/lateral fQRS associated with a higher risk of CV (adjusted HR: 1.44 [1.02, 2.05] and $2.00[1.26,3.16]$ for inferior and anterior/lateral fQRS, respectively), and all-cause mortality (adjusted HR: $1.35[1.04,1.74]$ and 1.84 $[1.32,2.56]$ for inferior and anterior/lateral fQRS, respectively) in multivariate model with adjustment for age, gender, body mass index, hypertension, diabetes mellitus, heart failure, hyperlipidemia, cardiovascular disease, estimated glomerular filtration rate, LV ejection fraction;. Kaplan-Meier survival curves for relevant clinical outcomes according to fQRS strata are displayed in Figure 5 (left) (all log-rank $p<0.001$ ). Clinical outcomes according to QRS duration strata (cutoff: $110 \mathrm{~ms}$ ) were detailed in Table S4, where risk stratification by QRS duration cutoff $(110 \mathrm{~ms})$ were less prominent compared to fQRS.

\section{Discussion}

In the present study, we examined the associations of QRS fragmentation utilizing standard 12-lead body surface ECGs in a large-scale HFpEF patient population and further related these measures to a variety of clinical endpoints, including HF hospitalization and death. We found that fQRS was not uncommon in HFpEF (31.6\%), with inferior fQRS nearly tripled the prevalence of anterior/inferior QRS. Despite comparable global LV ejection fractions, HFpEF patients manifesting fQRS, particularly those anterior/lateral fQRS, were associated with a more diseased myocardium, including distinct yet larger areas of myocardial perfusion defect, more unfavorable cardiac systolic/diastolic properties, and a higher prevalent coronary slow flow than those without fQRS. Anterior/lateral fQRS was further associated with a higher risk of HF hospitalization, with all HFpEF presenting with fQRS associated with a higher rate of mortality events during follow-up.

\section{Fragmentation of the QRS Complex}

Boineau and Cox (20) first demonstrated the occurrence of fractionated electrograms within ischemic regions using an experimental canine heart model after acute ischemia (21). Autopsy findings in patients with myocardial infarction have confirmed that islands of viable myocardial tissue interspersed in abundant fibrous tissue within myocardial necrotic regions may generate depolarized and depressed action potential upstroke velocities, resulting in slower electrical activation (22). This feature, termed fQRS phenotype by ECG, is responsible for inhomogeneous activation and altered depolarization of the ventricles and duplicate features of left BBB complicated by HF (23-25). Higher myocardial perfusion defects in those with known CAD along with an impaired coronary flow reserve in non-CAD HFpEF patients likely represented a higher total burden of macro/microvascular dysfunction, resulting in more 
extensive myocardial fibrosis and perturbed myocardial electrical spread. Notably, markedly diminished systolic and early myocardial relaxation velocities ( $s^{\prime}$ and $e^{\prime}$ ) representing overall more deteriorated intrinsic cardiac mechanics were observed when anterior/lateral fQRS existed in HFpEF patients. Our finding was in accordance with another recent work with a relatively small number of patients utilizing speckle-tracking based techniques (11). Higher BNP, higher troponin I levels and more unfavorable LV remodeling in our HFpEF patients complicated by fQRS likely represents higher LV wall stress, excessive extracellular matrix turnover/fibrotic replacement, and more extensive myocardial involvement (26)

\section{Relationships Between fQRS and Cardiac Outcomes}

The prognostic value of QRS fragmentation during acute coronary syndrome has been well demonstrated $(27,28)$. fQRS was prevalent in up to $30 \%$ of CAD subjects manifesting preserved LV function, with those presenting fQRS demonstrating a more dilated LV dimension, lower LVEF, higher wall motion abnormality, and reduced global 2D circumferential strains as compared with those without fQRS (29). In another study, the presence of fQRS in a healthy population was associated with reduced LV global longitudinal strain compared to those without (11). However, these two studies were limited by their small sample size and lack of cardiac outcomes. In one large epidemiological study conducted among 10904 individuals from the Finnish general population, fQRS was prevalent in $19.7 \%$ of subjects, including $15.7 \%$ in inferior leads, $0.8 \%$ in lateral leads, and $2.9 \%$ in anterior leads (12), with presence of lateral fQRS rather than anterior or inferior fQRS associated with higher all-cause, arrhythmic, and cardiac deaths. Interestingly, the presence of fQRS also conferred a higher risk of ventricular tachyarrhythmia in hypertrophic cardiomyopathy individuals (30). Notably, our data also showed that morphological fQRS phenotypes may serve as better functional and survival indicator for HFpEF compared to QRS duration (cutoff: $110 \mathrm{~ms}$ ). Our study is the first and, thus far, the largest to show the prevalence of fQRS in HFpEF patients ( 31.6\%), with inferior fQRS being more common than anterior/lateral fQRS, though the presence of anterior/lateral fQRS (8.6\%) was associated with a higher HF re-hospitalization rate all-cause mortality.

The condensed illustrations of the three fQRS strata about the clinical features, functional correlates, and outcomes in patients with HFpEF was demonstrated in Figure 5 (right).

\section{Limitations}

Our study has several limitations. Firstly, our findings may be limited by the retrospective study design. Second, myocardial perfusion defects, as assessed by the SPECT method, did not clarify the exact underlying causes, for example, irreversible myocardial damage (such as scar formation), residual incomplete re-vascularization, or restenosis in smaller caliber coronary vessels after intervention. Third, the causal relationship between QRS fragmentation and the observed perfusion defect cannot be ascertained, as it has been reported that disorganized myocardial electrical conduction through the myocardium may, on the other hand, result in an abnormal myocardial perfusion pattern. Despite these limitations, our findings demonstrated that patients manifesting QRS fragmentation may represent a specific HFpEF phenotype of undertreated macro/microvascular consequences from multiple comorbidities (such as hypertension, diabetes, and coronary artery disease) leading to regional 
myocardial dyskinesia (31). Further advanced functional and myocardial characterization imaging using magnetic resonance imaging may be more helpful in future studies to solve these issues.

\section{Conclusion}

The presence of fQRS in HFpEF, especially those manifesting anterior or lateral fQRS, was associated with more severe involvement of cardiac damage. These may include a more extensive deficit in myocardial perfusion and worsened myocardial functional properties, which likely translate into more unfavorable clinical outcomes. Early recognition of such a patient population may be helpful for more intensive targeted therapeutic interventions.

\section{Declarations}

\section{Grants}

This research was supported by the Ministry of Science and Technology (Taiwan) (grants NSC-101-2314B-195-020, NSC103-2314-B-010-005-MY3, 103-2314-B-195-001-MY3, 101-2314-B-195 -020 -MY1, MOST 103-2314-B-195-006-MY3, NSC102-2314-B-002-046-MY3, and 106-2314-B-195 -008 -MY2), MacKay Memorial Hospital $(10271,10248,10220,10253,10375,10358, E-102003)$, the Taiwan Foundation for Geriatric Emergency and Critical Care.

\section{References}

1. Das, M. K., Khan, B., Jacob, S., Kumar, A. \& Mahenthiran, J. Significance of a fragmented QRS complex versus a Q wave in patients with coronary artery disease. Circulatio.113,2495-2501(2006)

2. Das, M. K. et al. Fragmented wide QRS on a 12-lead ECG: A sign of myocardial scar and poor prognosis. Circ Arrhythm Electrophysiol, 1, 258-268 (2008).

3. Fares, H. et al. Fragmented QRS complexes-a novel but underutilized electrocardiograhic marker of heart disease. Crit Pathw Cardiol, 12, 181-183 (2013).

4. Das, M. K., Zipes, D. P. \& Fragmented, Q. R. S. a predictor of mortality and sudden cardiac death. Heart Rhythm. 6, Supplement:S8-14(2009)

5. Schuller, J. L. et al. Electrocardiographic characteristics in patients with pulmonary sarcoidosis indicating cardiac involvement. J Cardiovasc Electrophysiol, 22, 1243-1248 (2011).

6. Torigoe, K. et al. The number of leads with fragmented QRS is independently associated with cardiac death or hospitalization for heart failure in patients with prior myocardial infarction. J Cardiol, 59, 36-41 (2012).

7. Das, M. K. et al. Fragmented QRS on a 12-lead ECG: A predictor of mortality and cardiac events in patients with coronary artery disease. Heart Rhythm, 4, 1385-1392 (2007).

8. Pourafkari, L., Ghaffari, S. \& Nader, N. D. Prognostic value of fragmented QRS on admission in nonST-elevation myocardial infarction. Ann Noninvas Electrocardiol, 22, e12344 (2017). 
9. Morita, H. et al. Fragmented QRS as a marker of conduction abnormality and a predictor of prognosis of Brugada syndrome. Circulation, 118, 1697-1704 (2008).

10. Pandey, A. et al. Temporal trends in prevalence and prognostic implications of comorbidities among patients with acute decompensated heart failure: the ARIC study community surveillance. Circulation, 142, 230-243 (2020).

11. Reza, D. M. et al. Fragmented QRS and subclinical left ventricular dysfunction in individuals with preserved ejection fraction: A speckle-tracking echocardiographic study. J Arrhythm, 36, 335-340 (2020).

12. Terho, H. K. et al. Prevalence and prognostic significance of fragmented QRS complex in middle-aged subjects with and without clinical or electrocardiographic evidence of cardiac disease. Am J Cardiol, 114, 141-147 (2014).

13. Ollitrault, P. et al. Prevalence and significance of fragmented QRS complex in lead V1 on the surface electrocardiogram of healthy athletes. Europace, 22, 649-656 (2020).

14. Chien, S. C. et al. Malnutrition in acute heart failure with preserved ejection fraction: clinical correlates and prognostic implications. Eur J Heart Fail, 6, 953-964 (2019).

15. Narayanan, K. et al. QRS fragmentation and sudden cardiac death in the obese and overweight. J Am Heart Assoc, 4, e001654 (2015).

16. Hung, C. L. et al. Dose-related ethanol intake, Cx43 and Nav1.5 remodeling: exploring insights of altered ventricular conduction and QRS fragmentation in excessive alcohol users. J Mol Cell Cardiol, 114, 150-160 (2018).

17. Lang, R. M. et al. Recommendations for cardiac chamber quantification by echocardiography in adults: an update from the American Society of Echocardiography and the European Association of Cardiovascular Imaging. J Am Soc Echocardiogr, 28, 1-39 https://doi.org/e14 (2015).

18. Nagueh, S. F. et al. Recommendations for the Evaluation of Left Ventricular Diastolic Function by Echocardiography: An Update from the American Society of Echocardiography and the European Association of Cardiovascular Imaging. J Am Soc Echocardiogr, 29, 277-314 (2016).

19. Gibson, C. M. et al. TIMI frame count: a quantitative method of assessing coronary artery flow. Circulation, 93, 879-888 (1996).

20. Boineau, J. P. \& Cox, J. L. Slow ventricular activation in acute myocardial infarction. A source of reentrant premature ventricular contractions. Circulation, 48, 702-713 (1973).

21. Gardner, P. I., Ursell, P. C., Fenoglio, J. J. J. \& Wit, A. L. Electrophysiologic and anatomic basis for fractionated electrograms recorded from healed myocardial infarcts. Circulation, 72, 596-611 (1985).

22. Friedman, P. L., Fenoglio, J. J. Jr. \& Wit, A. L. Time course for reversal of electrophysiological and ultrastructural abnormalities in subendocardial Purkinje fibers surviving extensive myocardial infarction in dogs. Circ Res, 36, 127-144 (1975).

23. Hatala, R. et al. Three distinct patterns of ventricular activation in infarcted human hearts. An intraoperative cardiac mapping study during sinus rhythm. Circulation, 91, 1480-1494 (1995). 
24. Wiener, I., Mindich, B. \& Pitchon, R. Endocardial activation in patients with coronary artery disease: effects of regional contraction abnormalities. Am Heart J, 107, 1146-1152 (1984).

25. Cate, T. J. F. T., Kelder, J. C., Plokker, H. W. M., Verzijlbergen, J. F. \& Hemel, N. M. V. Myocardial perfusion SPECT identifies patients with left bundle branch block patterns at high risk for future coronary events. J Nucl Cardiol, 17, 216-224 (2010).

26. Thawabi, M. et al. Cardiac troponin and outcome in decompensated heart failure with preserved ejection fraction. Cardiovasc Diagn Ther, 7, 359-366 (2017).

27. Das, M. K. et al. Usefulness of fragmented QRS on a 12-lead electrocardiogram in acute coronary syndrome for predicting mortality. Am J Cardiol, 104, 1631-1637 (2009).

28. Pietrasik, G., Goldenberg, I., Zdzienicka, J., Moss, A. J. \& Zareba, W. Prognostic significance of fragmented QRS complex for predicting the risk of recurrent cardiac events in patients with Q-wave myocardial infarction. Am J Cardiol, 100, 583-586 (2007).

29. Yan, G. H. et al. Subclinical left ventricular dysfunction revealed by circumferential 2D strain imaging in patients with coronary artery disease and fragmented QRS complex. Heart Rhythm, 9, 928-935 (2012).

30. Kang, K. W. et al. Fragmented QRS as a candidate marker for high-risk assessment in hypertrophic cardiomyopathy. Heart Rhythm, 11, 1433-1440 (2014).

31. Hedeer, F. et al. To what extent are perfusion defects seen by myocardial perfusion SPECT in patients with left bundle branch block related to myocardial infarction, ECG characteristics, and myocardial wall motion? J Nucl Cardiol, https://doi.org/10.1007/s12350-020-02180-7 (2020).

\section{Figures}



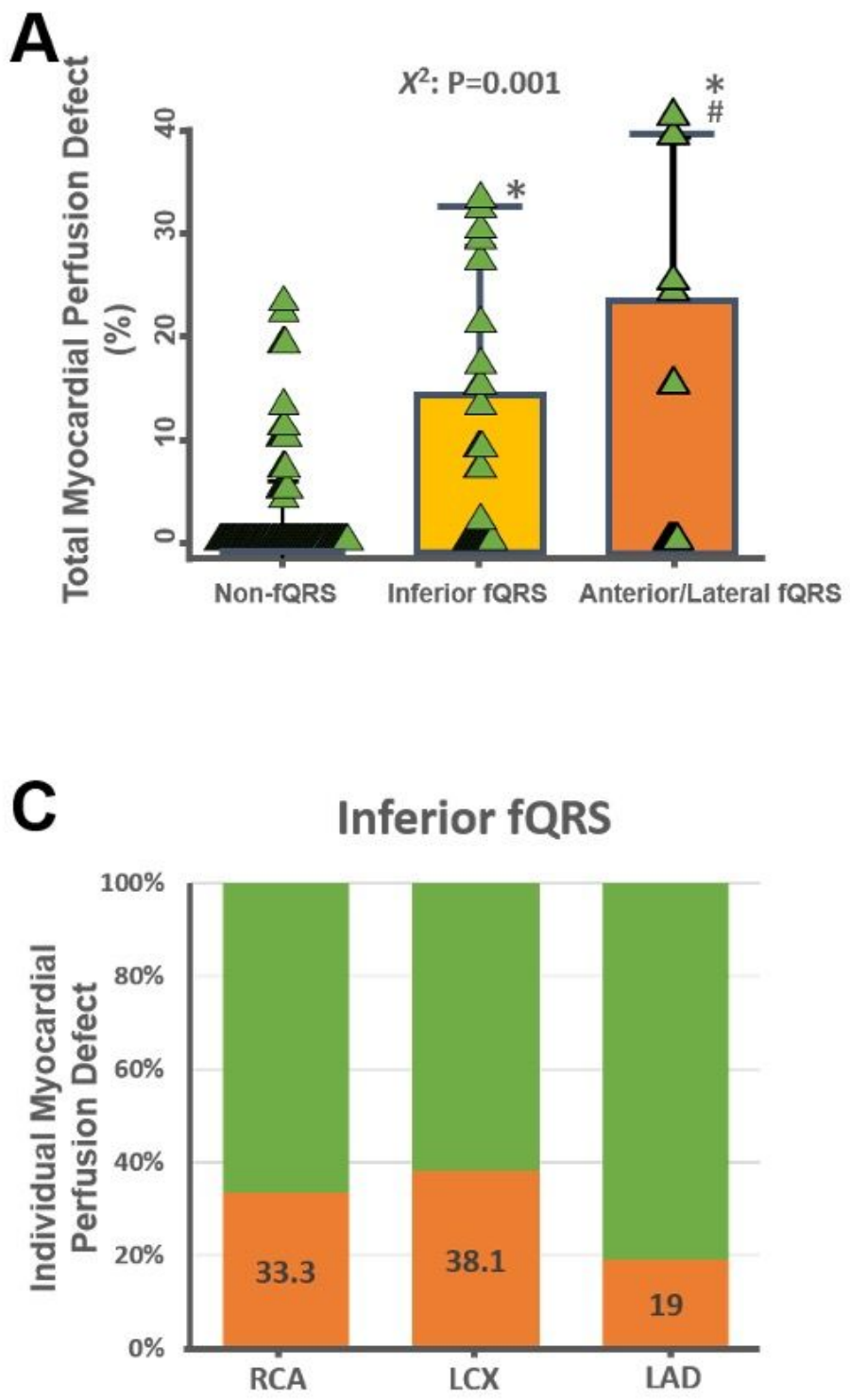

B

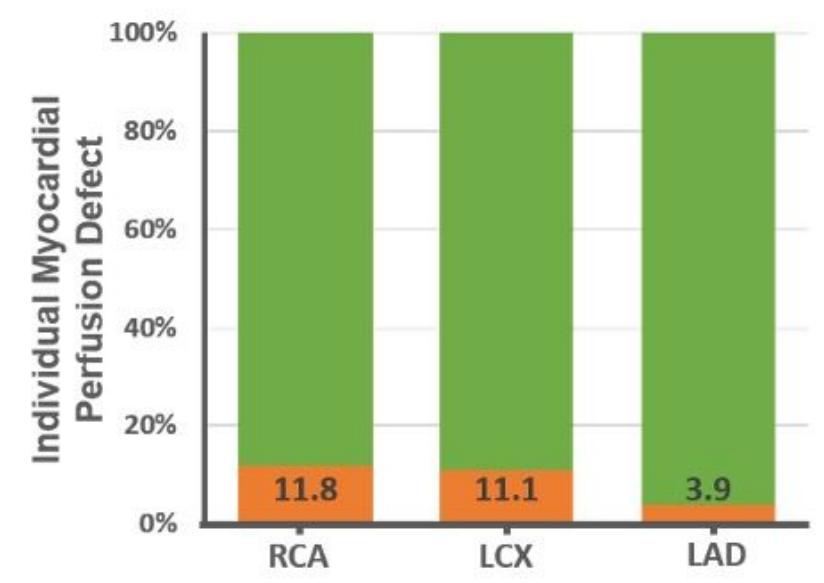

D

Anterior/Lateral fQRS

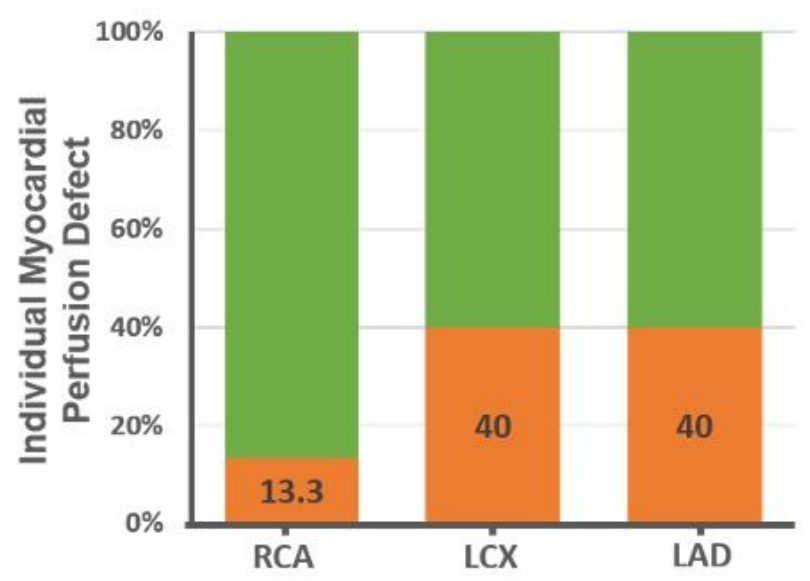

\section{Figure 1}

(A) The percentage of total myocardial perfusion defects in non-fQRS, inferior and anterior/lateral fQRS among 210 out of $326(64.4 \%)$ study participants with known CAD. (B-D) The percentage of myocardial perfusion defects in the RCA, LCX, and LAD in non-fQRS, inferior and anterior/lateral fQRS. RCA: right coronary artery, LCX: left circumflex artery, LAD: left anterior descending artery. 

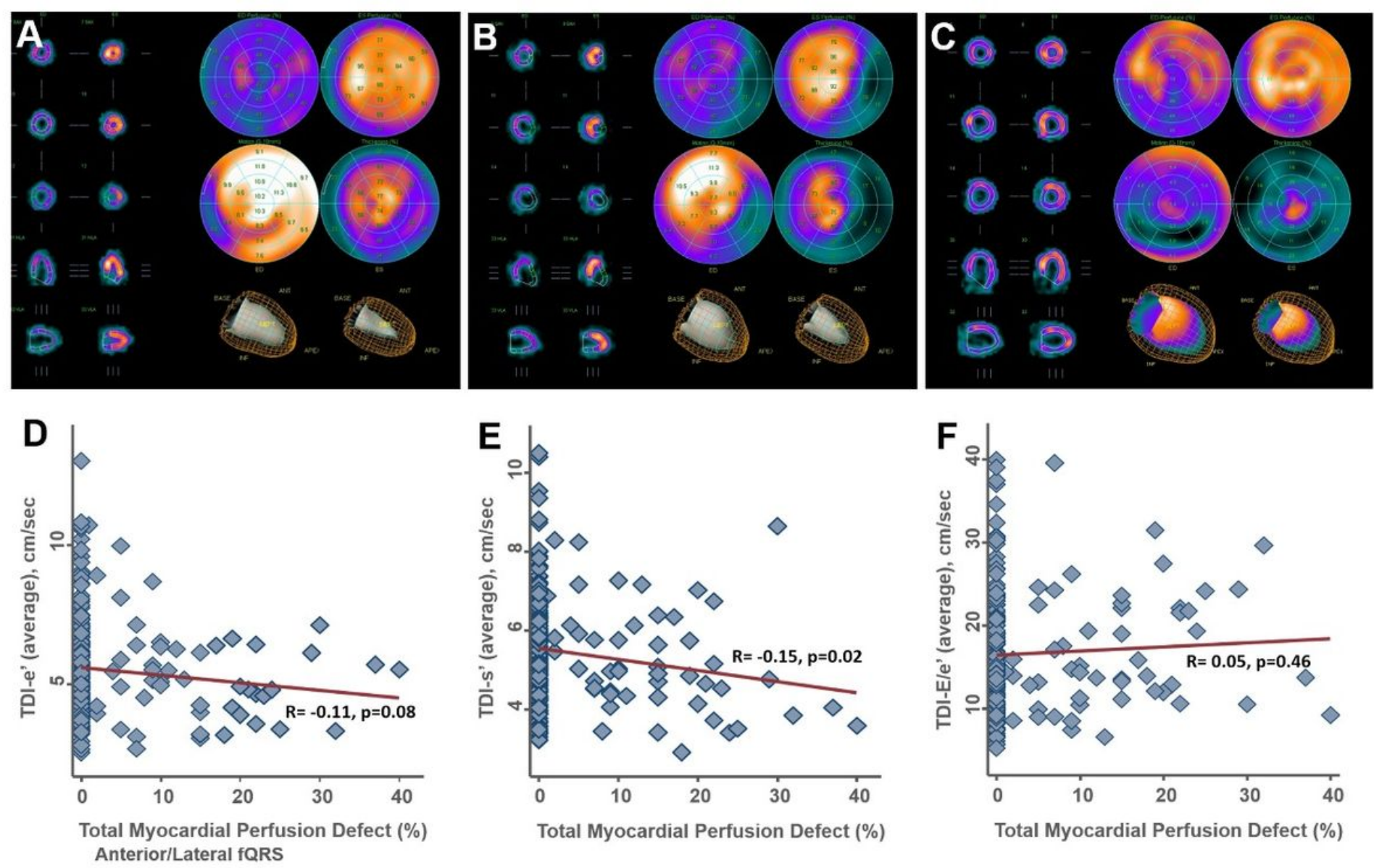

Figure 2

Illustration of myocardial perfusion defects in (A) non-fQRS, (B) inferior fQRS, and (C) anterior/lateral fQRS with SPECT imaging. (D-F) Correlation between total myocardial perfusion defect and TDI derived myocardial early relaxation (TDI-e') velocity, systolic (TDI-s') velocities and LV filling pressure E/e'. 
A

Slow Flow during Coronary Angiography ( $n=203$ )

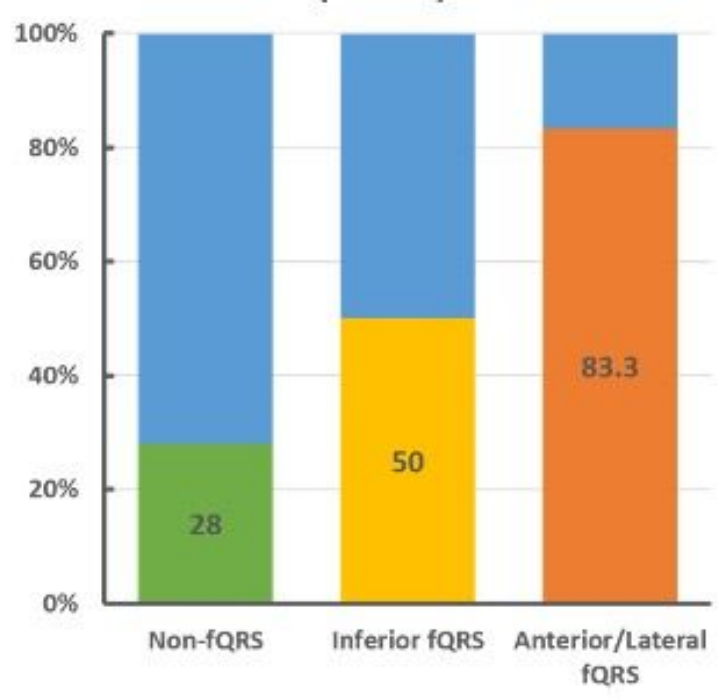

C

TDI-e' (average), cm/sec

$6.34 \pm 1.85$

$4.86 \pm 1.89$
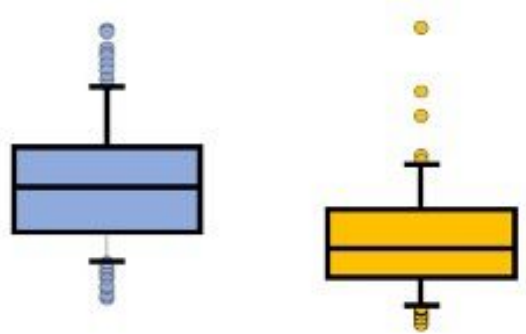

Slow Flow (-) Slow Flow (+)
B
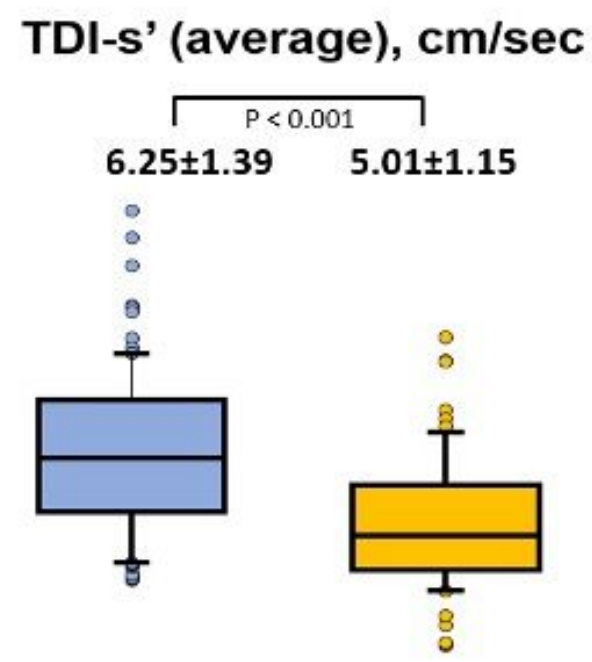

Slow Flow (-) Slow Flow (+)

D
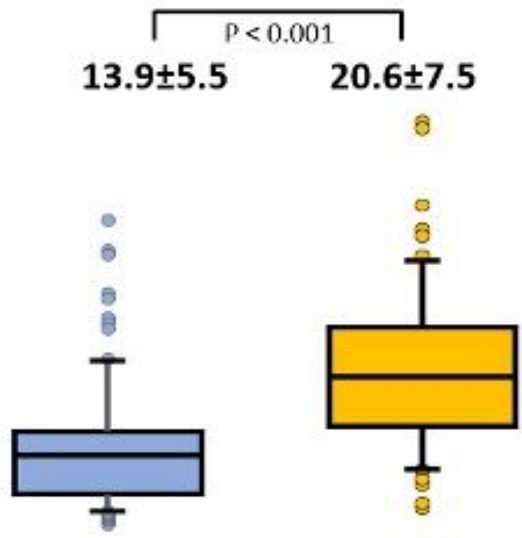

อ

Slow Flow (-) Slow Flow (+)

Figure 3

(A) The percentage of coronary slow flow during coronary angiography among 203 out of 634 (32.0\%) non-CAD study participants. (B-D) Comparisons of the TDI-s', TDI-e', and average E/e' in normal versus slow coronary flow non-CAD study participants. 


\section{A. Crude Model}

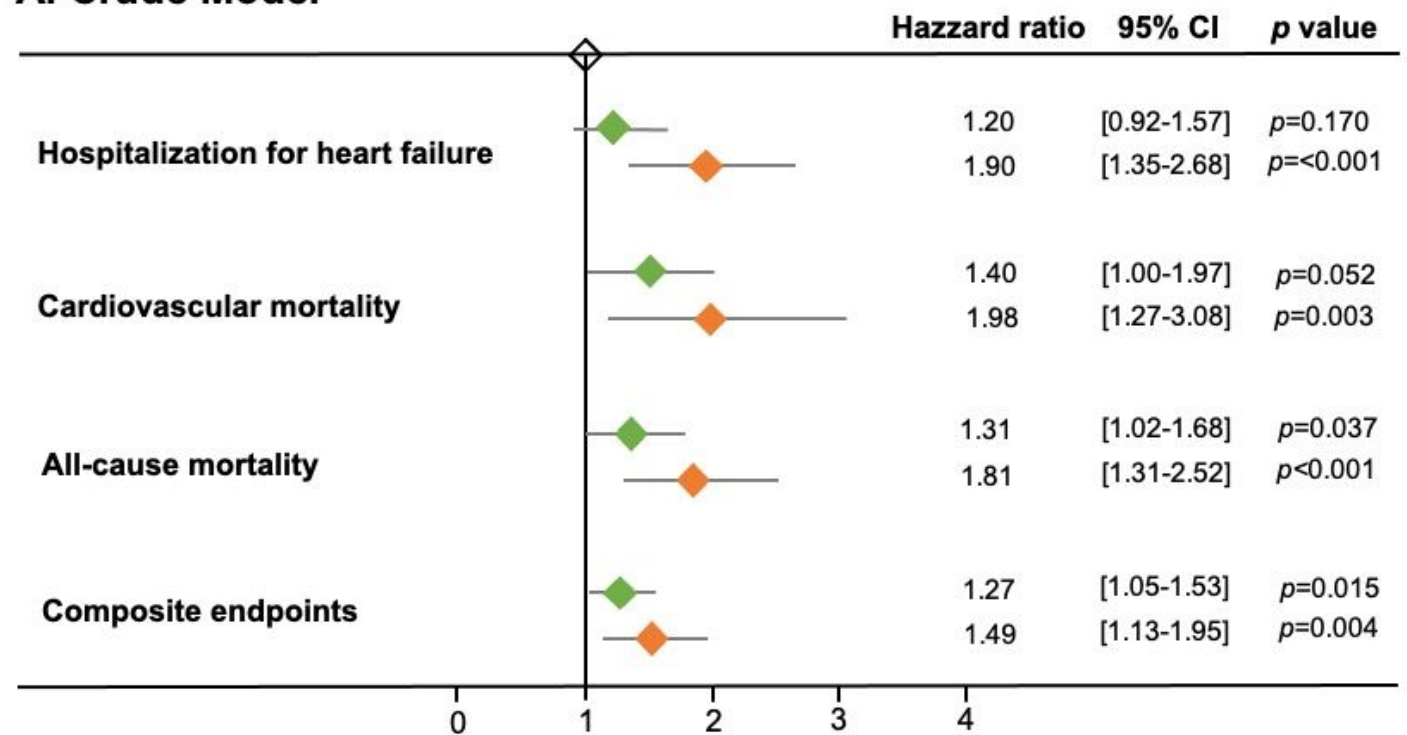

\section{B. Multivariate Model}

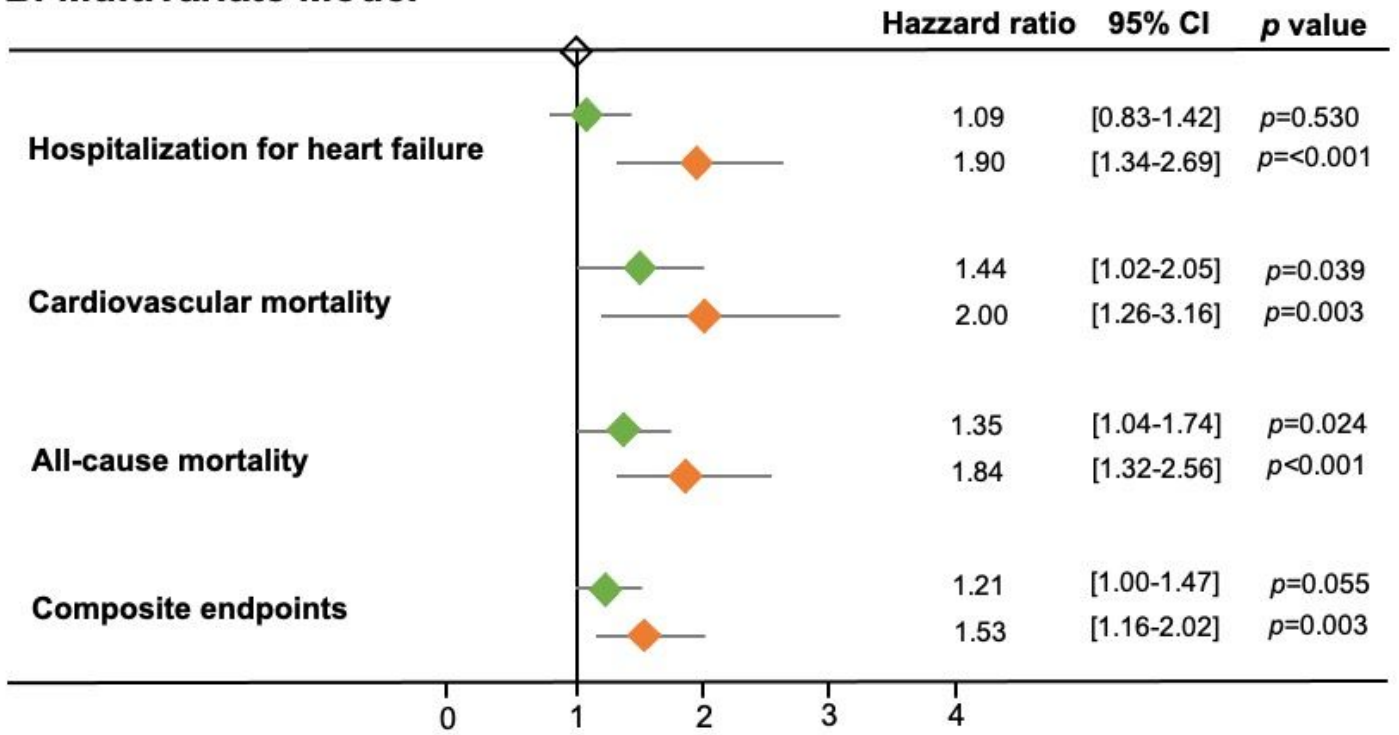

Non-fQRS (reference) Inferior fQRS Anterior/lateral fQRS $95 \%$ confidence interval

\section{Figure 4}

Crude and Adjusted Hazard ratio with corresponding 95\% confidence intervals (Cls) for 3 fQRS strata (non-fQRS as the reference) on hospitalization for heart failure, cardiovascular mortality, all-cause mortality, and composite endpoint. The multivariate model was adjusted for age, gender, body mass index, hypertension, diabetes mellitus, heart failure, hyperlipidemia, cardiovascular disease, estimated glomerular filtration rate, and left ventricular ejection fraction. 

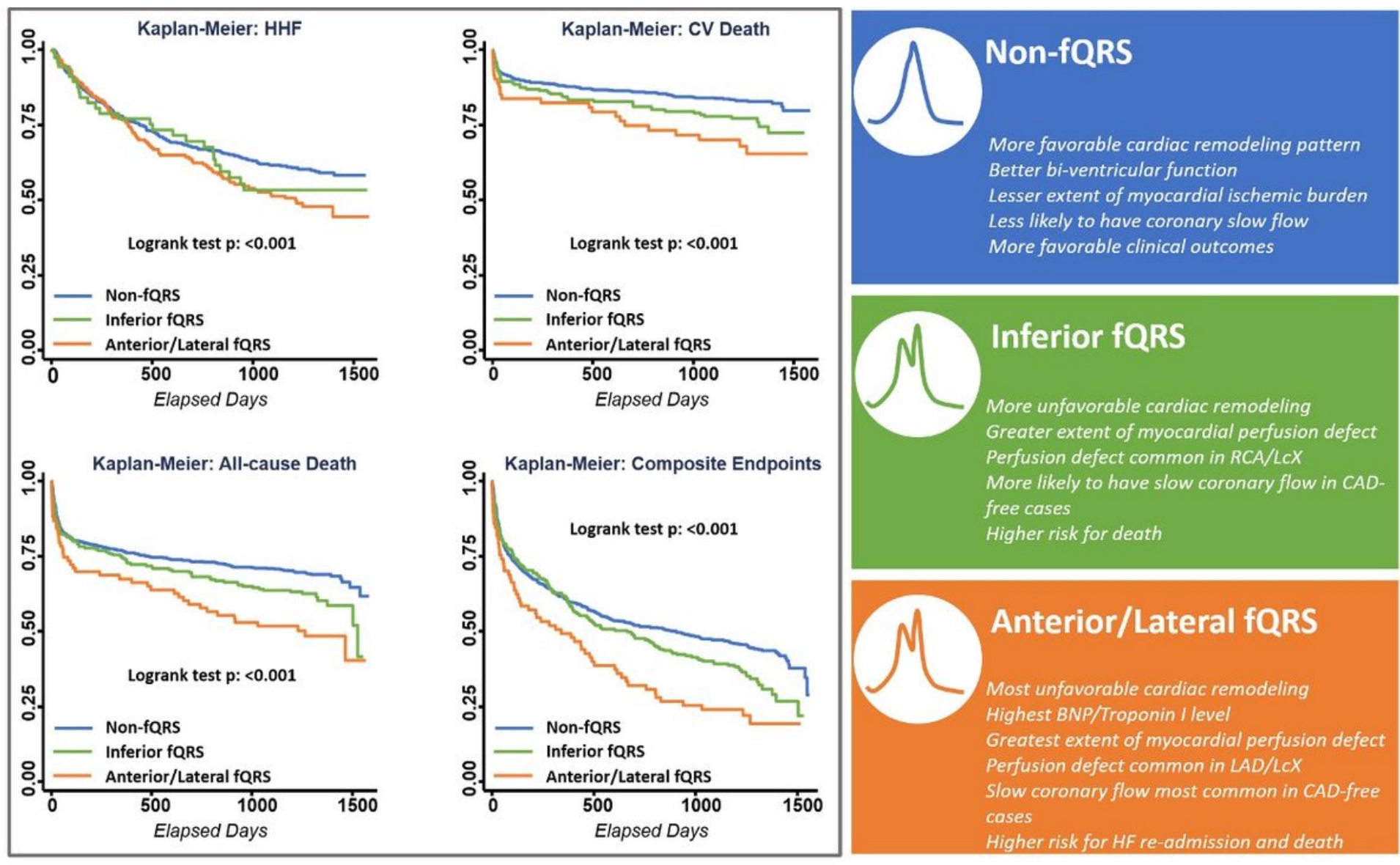

\section{Figure 5}

Kaplan-Meier survival curves of clinical outcomes according to fQRS strata (left). Condensed illustrations and graphical abstract about the clinical features, functional correlates, and outcomes of 3 fQRS strata in HFpEF (right).

\section{Supplementary Files}

This is a list of supplementary files associated with this preprint. Click to download.

- Supplementaryfigure1.png

- Supplementarymaterial.docx 